(CASE STUDY)

\title{
Successful use of topical nitroglycerine and warm compress for peripheral ischemia following arterial blood sampling in a neonate
}

\author{
Mohammad Reza Zarkesh 1,2,* \\ ${ }^{1}$ Maternal, Fetal and Neonatal Research Center, Tehran University of Medical Sciences, Tehran, Iran \\ 2 Department of Neonatology, Yas Women Hospital, Tehran University of Medical Sciences, Tehran, Iran.
}

Publication history: Received on 09 August 2020; revised on 23 August 2020; accepted on 26 August 2020

Article DOI: https://doi.org/10.30574/wjarr.2020.7.2.0296

\begin{abstract}
Although arterial catheter insertion is undertaken easily and safely, some serious complications like vasospasm, obstruction of the artery by thrombus or emboli have been reported. In the present study, a hospitalized case was reported with peripheral ischemia secondary to the brachial artery catheterization that successfully was treated with topical nitroglycerin $2 \%$ and warm compress. An early term neonate was born by the cesarean section with the first minute Apgar score 9. Immediately after birth, the subject was transferred to the NICU because of respiratory distress syndrome and subcostal retractions. Determining arterial blood gas, arterial blood sampling was ordered. Simultaneously with inserting a scalp vein in the right cubital fossa of the upper extremity, 4 fingers discolored to pale and white. Immediately scalp vein needle was removed and topical nitroglycerin $2 \%$ ointment was used while a warm compress was applied to the contralateral upper extremity. Gradual improvement in color and blood perfusion was observed in the fingers. One hour later, the white discoloration completely disappeared. Nitroglycerine $2 \%$ and warm compress to the contralateral extremity as safe and convenient measures could improve blood flow to peripheral ischemic tissues. However, in some cases more invasive pharmacological managements may be needed.
\end{abstract}

Keywords: Nitroglycerine; Warm compress; Peripheral ischemia; Catheterization

\section{Introduction}

Arterial blood sampling and cannulation in neonatal intensive care units are usually carried out through an arterial catheter insertion. The radial, ulnar, and posteriortibial arteries are common insertion sites. The arterial diameter, presence of sufficient circulation and collateral vessels should be considered priorto the insertion procedure [1]. Although arterial catheter insertion is undertaken easily and safely, some serious complications like vasospasm, obstruction of the artery by thrombus or emboli have been reported. Peripheral vasospasm by the frequency of about $20 \%$ may cause tissue ischemia. Pale or cyanotic discoloration, limitation of motion, absent pulses, coldness, and loss of extremity are some related complications $[2,3]$.

Preventing such potential serious risks, several conservative and pharmacological managements have been recommended. Immediate removal of the catheter, the elevation of the affected limb, application of a warm compress to the contralateral extremity, usage of topical vasodilators like nitroglycerin or administration of thrombolytic and anticoagulation agents such as tissue plasminogen activator and heparin are proposed treatments $[1,4-6]$.

In the present study, a NICU hospitalized case was reported with peripheral ischemia secondary to the brachial artery catheterization that successfully was treated with topical nitroglycerin $2 \%$ and warm compress to the contralateral extremity.

\footnotetext{
* Corresponding author Mohammad Reza Zarkesh

Maternal, Fetal and Neonatal Research Center, Tehran University of Medical Sciences, Tehran, Iran
} 


\section{Case Presentation}

An earlyterm female neonate with gestational age $37+1$ weeks and birth weight $2775 \mathrm{~g}$ was born to a 35 -year-old mother. The numbers of mother's gravid, pariety and abortion were 3,1 and 1, respectively. The biophysical profile score at 37 weeks was 8 of 8 and the amniotic fluid index was $5 \mathrm{Cm}$. Regarding the mother's obstetrical history, there was no history of gestational diabetes, hypertensive disorder, preeclampsia, convulsion, asthma and respiratory complication, thyroid, and thrombophilic disorders. At the admission time in the labor room, Nitrazin and the Amnisure ROM tests were negative and there were no complaints associated with vaginal discharge, reduction or absence of fetal movement. The newborn was born by cesarean section due to the breech presentation and oligohydramnios on 22 April 2020 at 9:30 Am with the first minute Apgar score 9. The birth height, chest, and head circumference were 57,36 , and $31 \mathrm{Cm}$, respectively. Immediately after birth, the subject was transferred to the neonatal intensive care unit (NICU) because of respiratory distress syndrome and subcostal retractions. Echocardiography examination was done and the findings showed patent ductus arteriosus (PDA), patent foramen ovale (PFO), and sub systemic pulmonary hypertension. Determining arterial blood gas, arterial blood sampling was ordered at 10:30 am. Simultaneously with inserting a scalp vein gauge 23 in the right cubital fossa of the upper extremity, 4 fingers discolored to pale and white (Figure 1).

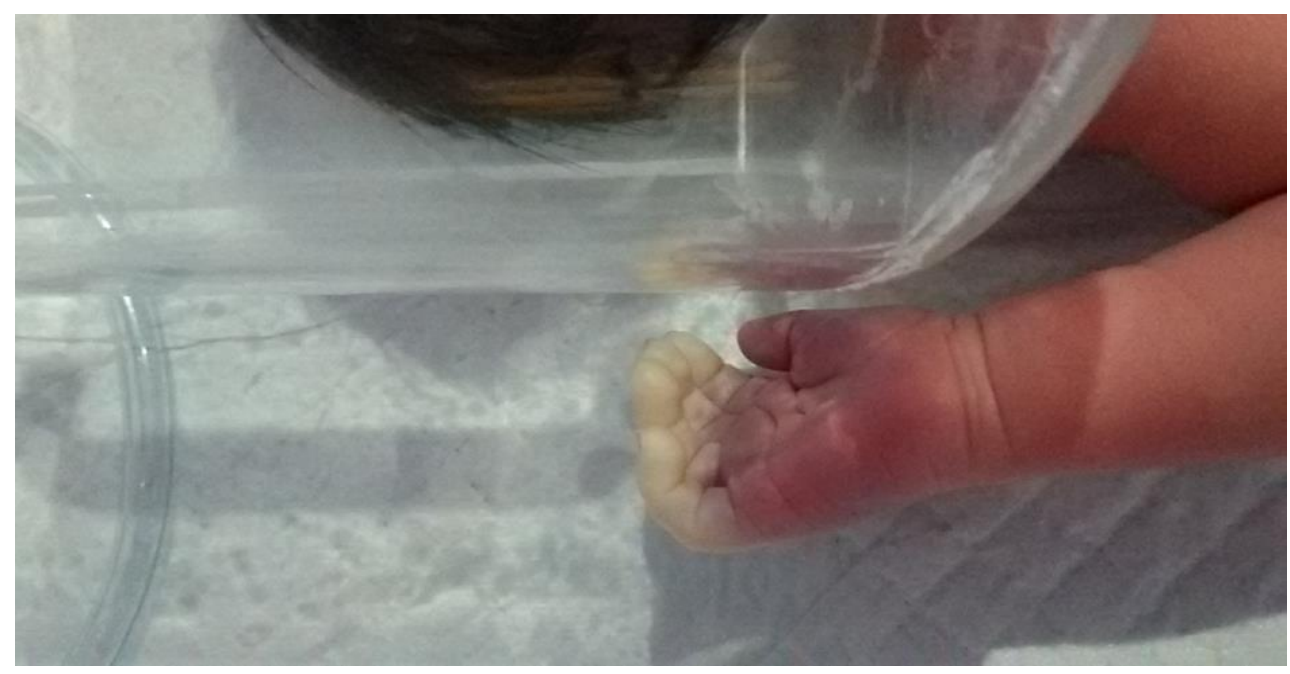

Figure 1Fingers discoloration following inserting of a scalp vein

Immediately scalp vein needle was removed and topical nitroglycerin $2 \%$ ointment was used while a warm compress was applied to the contralateral upper extremity. Gradual improvement in color and blood perfusion was observed in the fingers and one hour later at 11:30 Am., the white discoloration completely disappeared (Figure 2).

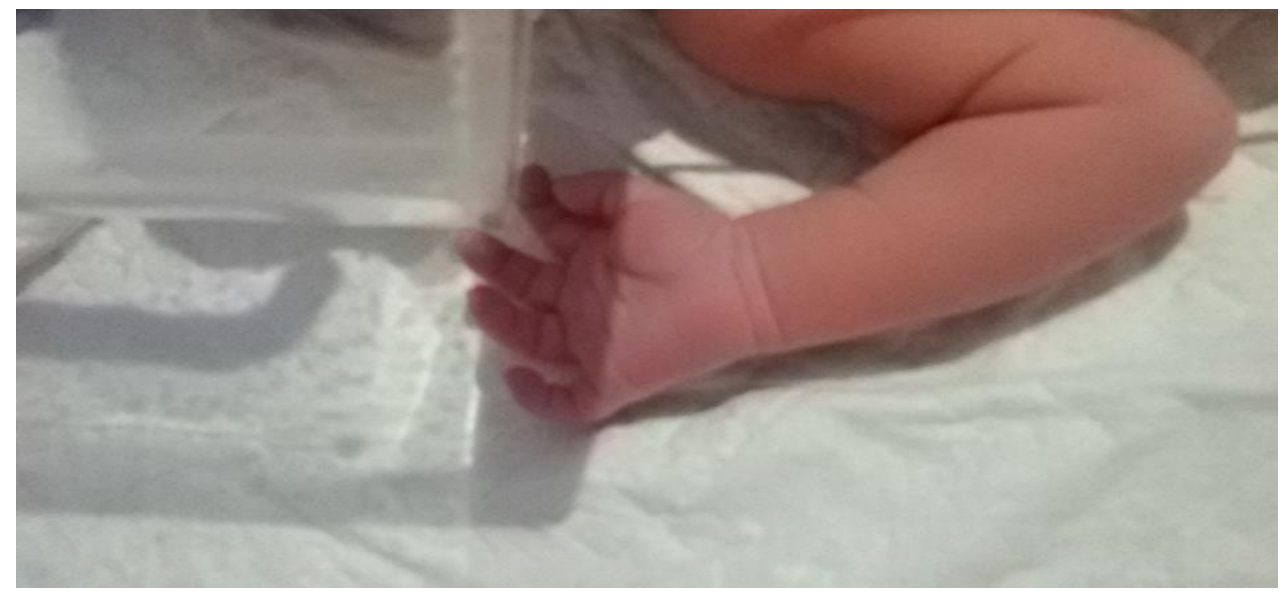

Figure 2 Gradual improvement after removal of scalp vein needle, use of topical nitroglycerin $2 \%$ ointment and warm compress on contralateral upper extremity 
No adverse effects related to the treatment were observed in the patient. Blood sampling was done from the left upper extremity without any complication. The results of the laboratory blood tests are demonstrated in Table 1.

Table 1 The results of arterial blood gas and routine laboratory blood tests.

\begin{tabular}{ll}
\hline ABG Results & 7.33 \\
$\mathrm{PH}$ & 39 \\
$\mathrm{PaCO}_{2}$ (mmHg) & 96 \\
$\mathrm{PaO}_{2}$ (mmHg) & 20.6 \\
$\mathrm{HCO}_{3}(\mathrm{meq} / \mathrm{L}$ ) & -4.9 \\
Base excess (mEq/L) & 0 Positive \\
Blood group & \\
Blood test & 120 \\
Blood sugar (mg/dL) & 0.8 \\
Creatinine (mg/mL) & 139 \\
Na (mmol/L) & 8.8 \\
Ca (mg/dL) & 4.3 \\
$\mathrm{~K}(\mathrm{mmol} / \mathrm{L})$ & 2.1 \\
Mg (mEq/L) & 0.2 \\
CRP (mg/L) & 3.1 \\
Retic (\%) & Normal \\
PBS & 17 \\
Hb (g/dL) & 49.2 \\
HCT (\%) & 326000 \\
Platelet (cells/mcL) & 102.9 \\
MCV (fL) & 13900 \\
WBC (cells/mcL) & 48 \\
Neutrophils (cells/mcL) & 2 \\
Eosinophils (cells/mcL) & 4 \\
Monocytes (cells/mcL) & 46 \\
Lymphocytes (cells/mcL) & \\
\hline
\end{tabular}

\section{Discussion}

In the case presented here, ischemic injury following brachial artery catheterization was diagnosed. Although brachial artery catheterization seems safe and easily accessible, we found that this procedure was not without risks; neonates with small arterial diameter are susceptible to some severe complications like digital or limb necrosis. Other studies have also reported some risks related to puncture of the brachial artery because of its proximity to the median nerve and the risk of thrombosis formation. Any mechanical stimulation of the vessels particularly in medium-sized arteries may cause smooth muscle contraction in the vessel walls. Furthermore, catheterization may damage the endothelium and intimae layer that stimulates inflammatory cascade resulting in arterial spasm, releasing vasoconstrictor agents, platelets aggregation, localized blood stasis, and tissue ischemia $[1,3,7]$.

We observed that tissue ischemia and fingers discoloration were alleviated with some simple treatments including immediate removal of scalp vein, application of topical nitroglycerin $2 \%$ ointment and warm compresses to the contralateral upper extremity. Nitroglycerin has been introduced in patients with acute pain associated with angina pectoris since 1879 [2]. Nitroglycerin as a nitric oxide component is well absorbed through intact skin. Nitroglycerin induces relaxation in vessels' smooth muscle and enhances collateral circulation. These mechanisms lead to arterial vasodilatation and blood flow improvement. The effects of nitroglycerin are positively correlated to the amount of ointment as well as the area of application. The onset of action is observed after 15-30 minutes and lasts to about 8 hours $[2,4,6]$. Moreover, we observed that applying a warm compress to the contralateral upper limb was beneficial. 
The application of a warm compress to the contralateral limb stimulates the autonomic nervous system resulting in reflex vasodilation [1]. A laser Doppler flowmetry investigation has demonstrated that a warm compress to the contralateral limb could increase the blood flow of the other limb [8]. Another study reported that warming a contralateral upper or lower extremity improved skin temperatures in a discolored lower extremity after umbilical arterial catheterization and there was no need for removal of catheter [9].

\section{Conclusion}

In summary, we reported the successful treatment of peripheral ischemia following arterial catheterization with nitroglycerine $2 \%$ and warm compress. However, it should be noticed that in some cases more invasive managements and pharmacological measures may be needed.

\section{Compliance with ethical standards}

\section{Acknowledgments}

I acknowledge the NICU colleagues for their kindly collaboration. This study was supported by Tehran University of Medical Sciences (TUMS).

\section{Disclosure of conflict of interest}

The authors declare that there is no conflict of interests.

\section{Statement of informed consent}

The parent of newborn signed an informed consent before entering the study.

\section{References}

[1] Mosalli R, Elbaz M andPaes B. (2013). Topical Nitroglycerine for Neonatal Arterial AssociatedPeripheral Ischemia following Cannulation: A Case Report and Comprehensive Literature Review. Case Rep Pediatr. 608516, 1-7.

[2] Baserga MC, Puri A and Sola A. (2002). The Use of Topical Nitroglycerin Ointment to Treat Peripheral Tissue Ischemia Secondary to Arterial Line Complications in Neonates. J Perinatol, 22, 416 - 9.

[3] Vasquez P, Burd A, Mehta R, Hiatt M andHegyi T. (2003). Resolution of Peripheral Artery Catheter-induced Ischemic Injury Following Prolonged Treatment with Topical Nitroglycerin Ointment in a Newborn: A Case Report. J Perinatol, 23, 348-50.

[4] Del Hoyo P, Ruiz P, del Rio M, Lopez-Menchero J and Garcia Cabezas M. (2016). Use of topical nitroglycerin in newborns with ischaemic injuries after vascular cannulation. AnPediatr (Barc), 85(3), 155-9.

[5] Olugbenga A, Dinesh S, Rodney S, Edwin F andMatei P. (2012). High-Dose Tissue Plasminogen Activator, Topical Nitroglycerin, and Heparin for Severe Ischemic Injury in a Neonate. ClP, 51(11), 1095-8.

[6] Wong AF, McCulloch L and Sola A. (1992). Treatment of peripheral tissue ischemia with topical nitroglycerin ointment in neonates. Clinical and laboratory observations. J. Pediatr, 121(6), 980-3.

[7] AkeLindbom. (1957). Arterial spasm caused by puncture and catheterization anarteriographic study of patients not suffering from arterial disease. ActaRadiol, 47(6), 449-460.

[8] Atherton DH andHoath S. (2007). Changes in skin blood flow over the foot with warming of the contralateral heel. ActaPædiatr, 87, 416-8.

[9] Blackmon LR. (1968). Treating neonatal vasospasm. Lancet, 2, 639. 